\title{
The Effects of Caffeine on Metabolomic Responses to Muscle Contraction in Rat Skeletal Muscle
}

\author{
Satoshi Tsuda ${ }^{1}$, Tatsuya Hayashi ${ }^{1}$ and Tatsuro Egawa ${ }^{1,2, * \mathbb{D}}$ \\ 1 Laboratory of Sports and Exercise Medicine, Graduate School of Human and Environmental Studies, \\ Kyoto University, Kyoto 606-8501, Japan \\ 2 Laboratory of Health and Exercise Sciences, Graduate School of Human and Environmental Studies, \\ Kyoto University, Kyoto 606-8501, Japan \\ * Correspondence: egawa.tatsuro.4u@kyoto-u.ac.jp; Tel.: +81-75-753-6613; Fax: +81-75-753-6885
}

Received: 4 July 2019; Accepted: 5 August 2019; Published: 7 August 2019

\begin{abstract}
Exercise has beneficial effects on our health by stimulating metabolic activation of skeletal muscle contraction. Caffeine is a powerful metabolic stimulant in the skeletal muscle that has ergogenic effects, including enhanced muscle power output and endurance capacity. In the present study, we aim to characterize the metabolic signatures of contracting muscles with or without caffeine stimulation using liquid chromatography-mass spectrometry and capillary electrophoresis coupled to mass spectrometry. Isolated rat epitrochlearis muscle was incubated in the presence or absence or of $3 \mathrm{mM}$ caffeine for $30 \mathrm{~min}$. Electrical stimulation (ES) was used to induce tetanic contractions during the final $10 \mathrm{~min}$ of incubation. Principal component analysis and hierarchical clustering analysis detected 184 distinct metabolites across three experimental groups-basal, ES, and ES with caffeine $(E S+C)$. Significance Analysis of Microarray identified a total of 50 metabolites with significant changes in expression, and 23 metabolites significantly changed between the ES and ES + C groups. Changes were observed in metabolite levels of various metabolic pathways, including the pentose phosphate, nucleotide synthesis, $\beta$-oxidation, tricarboxylic acid cycle, and amino acid metabolism. In particular, D-ribose 5-phosphate, IMP, O-acetylcarnitine, butyrylcarnitine, L-leucine, L-valine, and L-aspartate levels were higher in the ES $+\mathrm{C}$ group than in the ES group. These metabolic alterations induced by caffeine suggest that caffeine accelerates contraction-induced metabolic activations, thereby contributing to muscle endurance performance and exercise benefits to our health.
\end{abstract}

Keywords: metabolome; skeletal muscle; exercise; muscle contraction; ergogenic effect

\section{Introduction}

Exercise contributes to health benefits by reducing the risk of several chronic diseases. These effects are partly attributed to metabolic alterations that occur in contracting skeletal muscles. Exercise enhances muscle insulin sensitivity and mitochondrial function by stimulating master metabolic regulators, such as $5^{\prime}$-AMP-activated protein kinase (AMPK), sirtuin 1, and peroxisome proliferator-activated receptor- $\gamma$ co-activator $1 \alpha$ [1,2]. Recent evidence has suggested that secreted myokines from contracting skeletal muscles have positive effects on metabolic disorders [3]. Therefore, it is accepted that muscle contraction-induced metabolic activation is a key factor for maintaining normal physical function.

Caffeine is a powerful metabolic stimulant in the skeletal muscle. In vitro caffeine treatment of the skeletal muscle promotes insulin-independent glucose transport [4-8], fatty acid oxidation [8,9], $\mathrm{Ca}^{2+}$ release from the sarcoplasmic reticulum [10,11], and mitochondrial biogenesis [12]. We have recently demonstrated that caffeine increases the maximal capacity of contraction-stimulated AMPK activation and glucose transport in rat skeletal muscles [6]. Additionally, caffeine is thought to be an important contributor to ergogenic effects in humans. Meta-analyses have shown that caffeine intake has positive 
effects on muscle power output and endurance performance [13-15]. These findings suggest that caffeine accelerates muscle contraction-induced metabolic activation, thereby contributing to exercise benefits toward health promotion. However, there are no observations investigating the overall effects of caffeine on muscle contraction-induced metabolic activation in the skeletal muscle.

Metabolomic techniques are useful tools for the investigation of complex metabolic responses to muscle contraction $[16,17]$. In the present study, we aim to characterize the metabolic signatures of contracting muscles with or without caffeine stimulation, using liquid chromatography-mass spectrometry (LC-MS) and capillary electrophoresis coupled to mass spectrometry (CE-MS) analysis.

\section{Materials and Methods}

\subsection{Animals}

Male Sprague-Dawley rats (150-160 g) were purchased from Shimizu Breeding Laboratories (Kyoto, Japan). Rats were fed a standard diet (Certified Diet MF; Oriental Koubo, Tokyo, Japan) with ad libitum water and were subjected to overnight fasting before the experiments. All animal-related protocols were performed in accordance with the Guide for the Care and Use of Laboratory Animals as adopted and promulgated by the National Institutes of Health (Bethesda, MD, USA) and were approved by the Animal Use Committee at Kyoto University Graduate School of Human and Environmental Studies.

\subsection{Muscle Treatment}

Muscles were treated as previously described [6]. Rats were killed by cervical dislocation without anesthesia, and the epitrochlearis muscles were removed and mounted on to an incubation apparatus with the tension set to $0.5 \mathrm{~g}$. The epitrochlearis muscle is composed predominantly of fast-twitch glycolytic fibers (60-65\% fast-twitch white, 20\% fast-twitch red, 15\% slow-twitch red) [18], but also has higher oxidative potential than the other fast-twitch muscle [19]. Moreover, it is a small and thin muscle that is suitable for in vitro incubation study. The muscles were pre-incubated in alpha-minimum essential medium (21444-05, nacalai tesque, Kyoto, Japan) containing $1.0 \mathrm{~g} / \mathrm{L}$ glucose supplemented with $1 \%$ penicillin/streptomycin for $40 \mathrm{~min}$ and then incubated in fresh medium in the presence or absence of $3 \mathrm{mM}$ caffeine for $30 \mathrm{~min}$. For tetanic contractions, the muscles were stimulated using an electric stimulator (SEN-3401; Nihon Koden, Tokyo, Japan) during the final $10 \mathrm{~min}$ of the incubation period (train rate, $1 / \mathrm{min}$; train duration, $10 \mathrm{~s}$; pulse rate, $100 \mathrm{~Hz}$; pulse duration, $0.1 \mathrm{~ms}$; voltage; $10 \mathrm{~V}$ ). Basal muscles were pre-incubated and incubated without contraction and caffeine treatment. All media were continuously gassed with $95 \% \mathrm{O}_{2} / 5 \% \mathrm{CO}_{2}$ and maintained at $37^{\circ} \mathrm{C}$.

\subsection{Metabolomic Analysis}

Metabolomic analysis was performed by LSI Medience Corporation (Tokyo, Japan). In brief, the muscle samples ( $\geq 50 \mathrm{mg}$ ) were homogenized using beads and suspended into $1 \mathrm{~mL}$ distilled water. They were then mixed with methanol $(2 \mathrm{~mL})$ and chloroform $(2 \mathrm{~mL})$ for $10 \mathrm{~min}$ at room temperature. After centrifugation at $1000 \times g$ for $15 \mathrm{~min}$, the supernatant was evaporated using nitrogen gas and dissolved with $10 \%$ acetonitrile aqueous solution $(200 \mu \mathrm{L})$. After adding internal standards, the samples were subjected to both LC-MS and CE-MS. All peak positions (retention time and $m / z$ ) and areas were calculated using Markeranalysis (LSI Medience, Tokyo, Japan). All peak areas were aligned into one data sheet, and the errors of peak intensities were corrected using internal standards. Noise peaks were deleted after comparison with the peaks detected in blank samples. The metabolites were identified by comparing the retention times and $m / z$ values with a standard dataset provided by LSI Medience Corporation.

\subsection{Data Analysis}

After applying autoscaling (mean-centered and divided by standard deviation of each variable), principal component analysis (PCA), significance analysis of microarray (SAM), hierarchical clustering 
analysis (HCA), and one-way ANOVA with Tukey's multiple comparison test were performed using the web-based metabolomic data processing tool MetaboAnalyst 4.0 (http://www.metaboanalyst.ca, Xia Lab, McGill University, Montreal, Canada). In PCA, a score plot of the first and second principal components was generated. HCA was performed to exhibit simultaneous clustering of metabolites and samples by Euclidean distance using Ward's method. Heat maps were generated by coloring the values of all data across their value ranges. False discovery rates (FDR) were calculated to reduce the risk of false positives by adjusted $p$ values. FDR $<0.05$ was defined as statistically significant.

\section{Results and Discussion}

\subsection{Pattern Recognition of Metabolites}

Metabolomic analysis detected 184 metabolites by LC-MS and CE-MS (Table S1). PCA is a statistical procedure that is used for feature extraction. Using PCA on the detected 184 metabolites, three groups, i.e., basal, electrical stimulation (ES), and ES with caffeine $(E S+C)$, were clearly distinguished on the principal component (PC) 2, although they were overlapped on PC1 (Figure 1). We previously demonstrated that ES in isolated rat skeletal muscles induces metabolic activation [20]. Likewise, the PCA results in this study indicated that ES-induced muscle contraction influences the metabolomic profile of the skeletal muscle. Furthermore, PCA plots in the ES + C group were more distant from basal than in the ES group (Figure 1), suggesting that caffeine accelerates ES-induced metabolic responses.

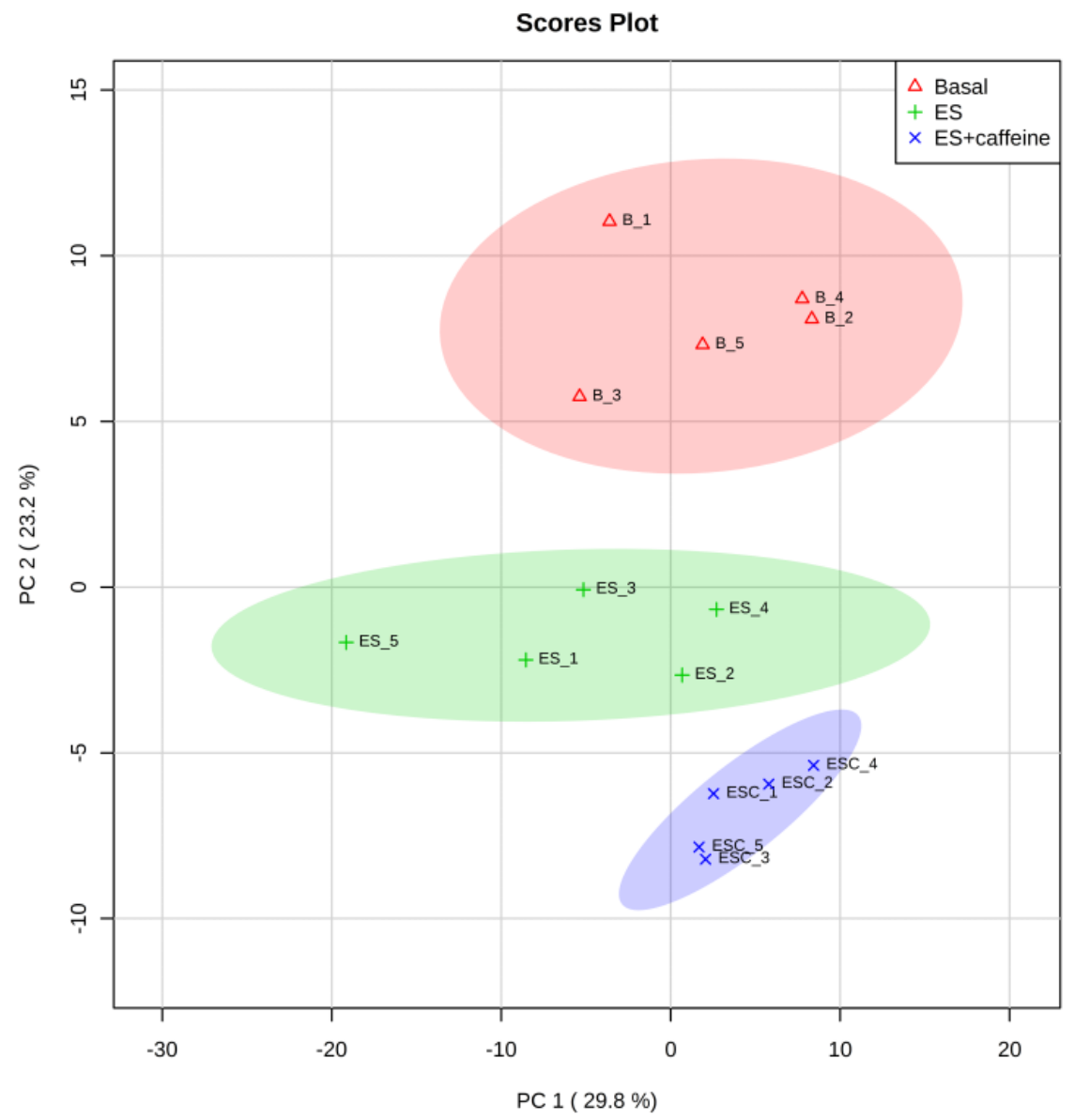

Figure 1. Principal component analysis (PCA) plot of the identified metabolites of the skeletal muscle of three groups, basal (B), electrical stimulation (ES), and electrical stimulation + caffeine (ESC). Principal components (PC1 and PC2) capture 53.0\% of the variation in the dataset. The elliptic areas represent the $95 \%$ confidence regions. 


\subsection{Discovery of Differentiating Metabolites}

To identify differentially expressed metabolites among the three groups, SAM, a popular method employed in microarray data analysis [21], was used. SAM identified a total of 50 metabolites with FDR $=0.007$ (Figure 2). Table 1 lists the identified compounds. HCA of the 50 metabolites showed that each group was tightly clustered and that the caffeine influenced the metabolite profiles of ES toward a high level of contents (Figure 3). Figures 4-7 show the 23 metabolites that significantly changed between the $\mathrm{ES}$ and $\mathrm{ES}+\mathrm{C}$ groups.

\section{SAM Plot for Delta $=1.3$}

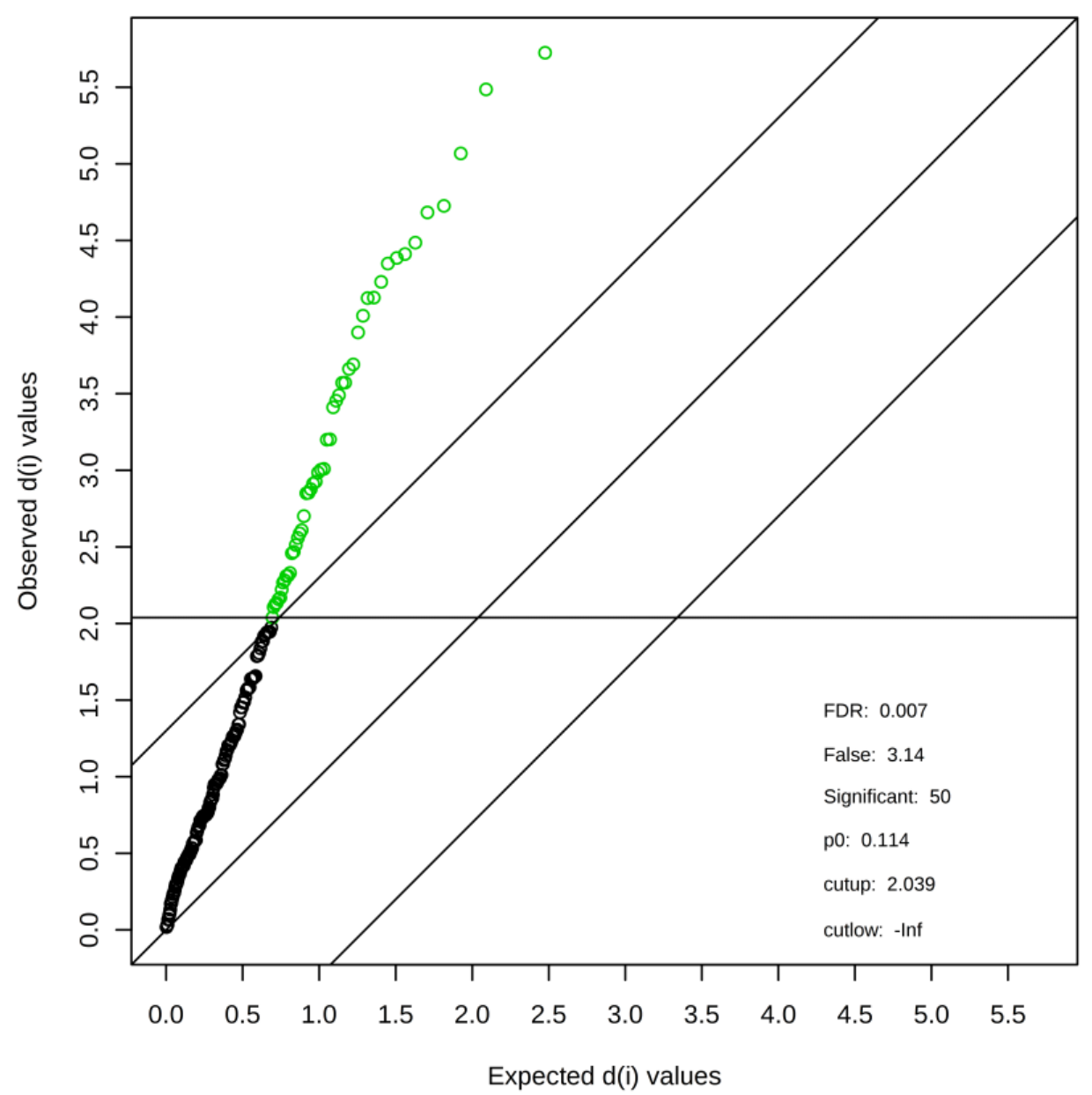

Figure 2. Identification of metabolites with significant changes in expression by significance analysis of microarray (SAM) among the three groups. The SAM plot is a scatter plot of the observed relative difference versus the expected relative difference. The solid diagonal line indicates where these two measures are the same. The dotted lines are drawn at a distance of delta from the solid line. The significant variables are highlighted in green and the details are shown in Table 1. 
Table 1. Significant features identified by SAM.

\begin{tabular}{|c|c|c|c|c|c|}
\hline & Name & d Value & SD & $p$ Value & q Value \\
\hline 1 & Theobromine & 5.7247 & 0.031023 & 0.0 & 0.0 \\
\hline 2 & Butyrylcarnitine (C4) & 5.4855 & 0.055814 & 0.0 & 0.0 \\
\hline 3 & IMP & 5.0676 & 0.10169 & 0.0 & 0.0 \\
\hline 4 & $\beta$-Aminoisobutyric acid & 4.7254 & 0.14193 & 0.0 & 0.0 \\
\hline 5 & L-Lactic acid & 4.6827 & 0.14713 & 0.0 & 0.0 \\
\hline 6 & Cytosine & 4.4855 & 0.1717 & $5.4348 \times 10^{-5}$ & $1.1445 \times 10^{-4}$ \\
\hline 7 & D-Ribose 5-phosphate & 4.4111 & 0.1812 & $5.4348 \times 10^{-5}$ & $1.1445 \times 10^{-4}$ \\
\hline 8 & D-Glyceraldehyde & 4.3854 & 0.18452 & $5.4348 \times 10^{-5}$ & $1.1445 \times 10^{-4}$ \\
\hline 9 & Hypoxanthine & 4.3489 & 0.18926 & $5.4348 \times 10^{-5}$ & $1.1445 \times 10^{-4}$ \\
\hline 10 & Maltotriose & 4.2295 & 0.205 & $5.4348 \times 10^{-5}$ & $1.1445 \times 10^{-4}$ \\
\hline 11 & D-Glucosamine 6-phosphate & 4.1269 & 0.21883 & $1.087 \times 10^{-4}$ & $1.7607 \times 10^{-4}$ \\
\hline 12 & Choline & 4.1237 & 0.21927 & $1.087 \times 10^{-4}$ & $1.7607 \times 10^{-4}$ \\
\hline 13 & Succinic acid & 4.0087 & 0.2351 & $1.087 \times 10^{-4}$ & $1.7607 \times 10^{-4}$ \\
\hline 14 & N4-Acetylcytidine & 3.8995 & 0.25048 & $1.6304 \times 10^{-4}$ & $2.4524 \times 10^{-4}$ \\
\hline 15 & L-Glutamate & 3.6907 & 0.28084 & $3.2609 \times 10^{-4}$ & $4.2917 \times 10^{-4}$ \\
\hline 16 & Acetylenedicarboxylate & 3.6613 & 0.28523 & $3.2609 \times 10^{-4}$ & $4.2917 \times 10^{-4}$ \\
\hline 17 & L-Leucine & 3.5719 & 0.29872 & $4.8913 \times 10^{-4}$ & $5.7223 \times 10^{-4}$ \\
\hline 18 & 1-Myristoylglycerophosphocholine & 3.5708 & 0.29889 & $4.8913 \times 10^{-4}$ & $5.7223 \times 10^{-4}$ \\
\hline 19 & L-Hexanoyl-carnitine (C6) & 3.4897 & 0.31135 & $5.4348 \times 10^{-4}$ & $6.0234 \times 10^{-4}$ \\
\hline 20 & Serotonin & 3.4538 & 0.31693 & $5.9783 \times 10^{-4}$ & $6.2945 \times 10^{-4}$ \\
\hline 21 & Threonate & 3.4109 & 0.32367 & $7.0652 \times 10^{-4}$ & $7.0847 \times 10^{-4}$ \\
\hline 22 & Glycerol-3-phosphate & 3.2018 & 0.35736 & 0.001413 & 0.0012937 \\
\hline 23 & 2-Oxobutanoate & 3.1997 & 0.35772 & 0.001413 & 0.0012937 \\
\hline 24 & ATP & 3.009 & 0.38981 & 0.0021739 & 0.0018047 \\
\hline 25 & N-Acetyl-L-alanine & 3.0039 & 0.3907 & 0.0021739 & 0.0018047 \\
\hline 26 & L-Alanine & 2.9853 & 0.39391 & 0.0022283 & 0.0018047 \\
\hline 27 & Xanthine & 2.9261 & 0.40421 & 0.0023913 & 0.001865 \\
\hline 28 & Imidazole lactic acid & 2.913 & 0.40651 & 0.0025543 & 0.001921 \\
\hline 29 & N8-Acetylspermidine & 2.8766 & 0.41292 & 0.002663 & 0.0019337 \\
\hline 30 & NADP & 2.8527 & 0.41718 & 0.0029348 & 0.0020305 \\
\hline 31 & Oxypurinol & 2.8504 & 0.41759 & 0.0029891 & 0.0020305 \\
\hline 32 & Taurine & 2.7011 & 0.44464 & 0.0040761 & 0.0026823 \\
\hline 33 & O-Acetylcarnitine (C2) & 2.6096 & 0.46169 & 0.0052174 & 0.0032651 \\
\hline 34 & Isatin & 2.5882 & 0.46572 & 0.0052717 & 0.0032651 \\
\hline 35 & L-Fucose & 2.5586 & 0.47133 & 0.0057065 & 0.0034334 \\
\hline 36 & Spermidine & 2.5126 & 0.48016 & 0.0064674 & 0.0037831 \\
\hline 37 & 1-Palmitoleoylglycerophosphocholine & 2.4683 & 0.48872 & 0.0071739 & 0.0040357 \\
\hline 38 & GDP & 2.4586 & 0.49062 & 0.0072826 & 0.0040357 \\
\hline 39 & Iminodiacetate & 2.3301 & 0.51606 & 0.0096196 & 0.0051941 \\
\hline 40 & L-Methionine & 2.3125 & 0.51962 & 0.010054 & 0.0052477 \\
\hline 41 & $\gamma$-Glu-leu & 2.3104 & 0.52004 & 0.010217 & 0.0052477 \\
\hline 42 & Maleamate & 2.2816 & 0.52587 & 0.010761 & 0.0052964 \\
\hline 43 & L-Aspartate & 2.2673 & 0.52879 & 0.010815 & 0.0052964 \\
\hline 44 & Guanidinosuccinic acid & 2.2214 & 0.53822 & 0.011793 & 0.0056442 \\
\hline 45 & L-Valine & 2.169 & 0.54911 & 0.013478 & 0.0062447 \\
\hline 46 & CDP & 2.1582 & 0.55136 & 0.013641 & 0.0062447 \\
\hline 47 & EDTA & 2.134 & 0.55645 & 0.014511 & 0.0064614 \\
\hline 48 & 2-Hydroxyisobutyric acid & 2.126 & 0.55815 & 0.014728 & 0.0064614 \\
\hline 49 & Creatinine & 2.1072 & 0.56212 & 0.015163 & 0.0065164 \\
\hline 50 & 3-Methyl-2-oxobutyric acid & 2.0385 & 0.57683 & 0.017065 & 0.0071872 \\
\hline
\end{tabular}




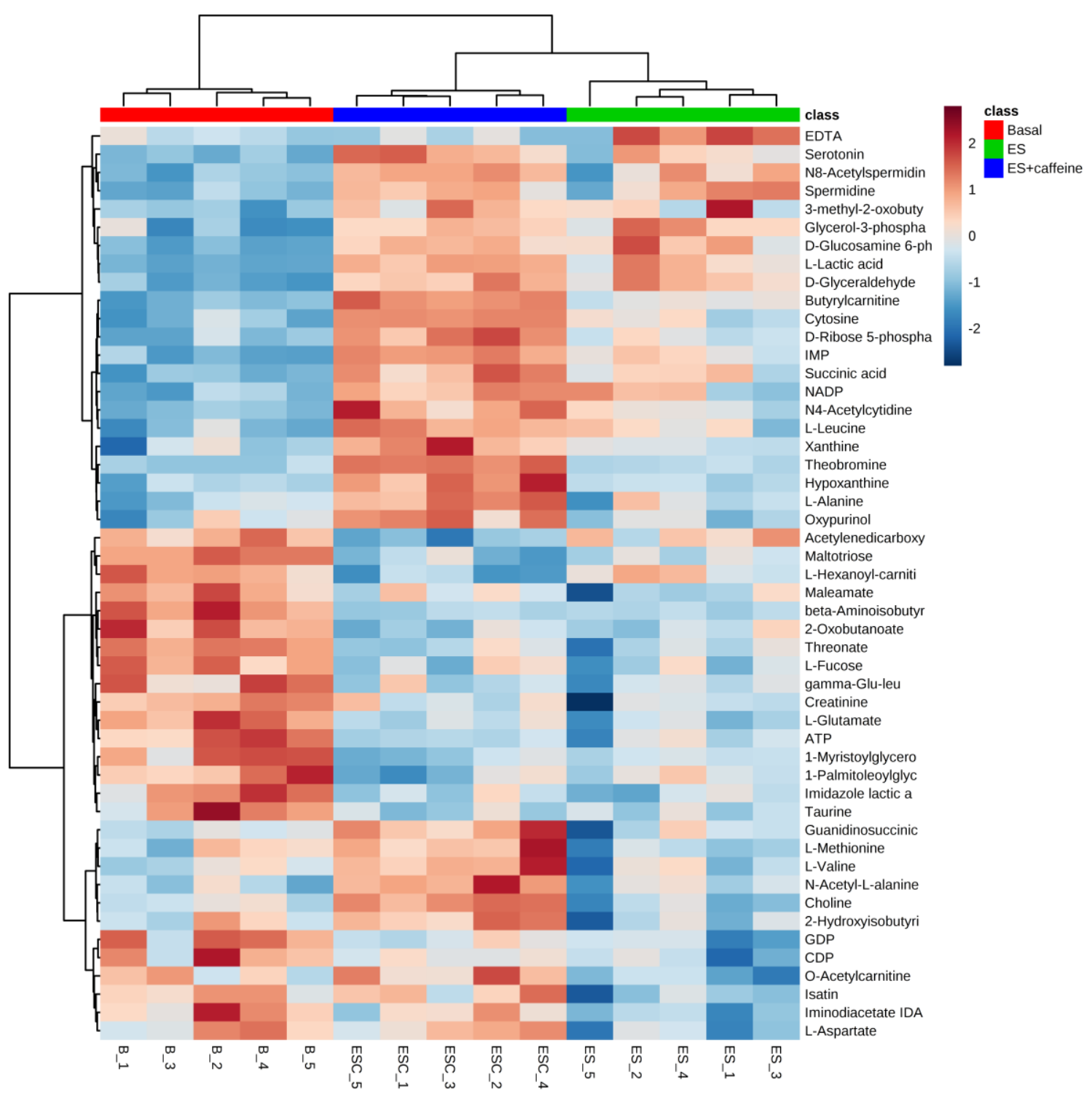

Figure 3. A heat map of hierarchical clustering analysis comparing the 50 different metabolites among groups. The heat map patterns among groups were distinguishable. The color red demonstrates that the relative content of metabolites is high and blue demonstrates that they are low.

\subsection{Pentose Phosphate Pathway/Nucleotide Synthesis Pathway}

The pentose phosphate pathway is an alternative pathway to glycolysis [22]. It does not lead to ATP formation, but rather, produces ribose 5-phosphate. Intracellular ribose 5-phosphate concentration is an important determinant of rates of de novo purine synthesis [23]. The synthesis of purine nucleotides begins with ribose 5-phosphate and produces the first fully formed nucleotide, IMP. IMP is accumulated in contracted skeletal muscle during exercise and accounted for ATP re-synthesis during recovery phase from exercise [24]. Therefore, pentose phosphate pathway activation and subsequent nucleotide synthesis is suggested to be important for maintaining cellular energy during and following exercise.

In the present study, ES increased D-ribose 5-phophate levels, and caffeine further increased this effect (Figure 4), indicating that caffeine promotes exercise-induced activation of the pentose phosphate pathway in skeletal muscles. It was also found that caffeine in conjunction with ES increased IMP levels, as compared to ES alone (Figure 4). Taken together, the stimulation of the pentose phosphate pathway by caffeine may contribute to recovery from energy depletion following muscle contraction by promoting ATP re-synthesis from IMP. In our previous study, caffeine alleviated muscle fatigue 
during contraction [6]. Furthermore, it has been suggested that activation of the pentose phosphate pathway stimulates energy production by enhancing mitochondrial function [25]. These metabolic responses may contribute to a positive effect of caffeine on endurance performance.

A small proportion of IMP is converted to inosine and further to hypoxanthine, and hypoxanthine is transformed to xanthine, which is then subsequently converted to uric acid and excreted in the urine [26]. In the present study, hypoxanthine and xanthine levels were increased by caffeine treatment in the contracted muscle (Figure 4), supporting the caffeine-induced accumulation of IMP.

\section{Pentose phosphate pathway/nucleotide synthesis pathway}
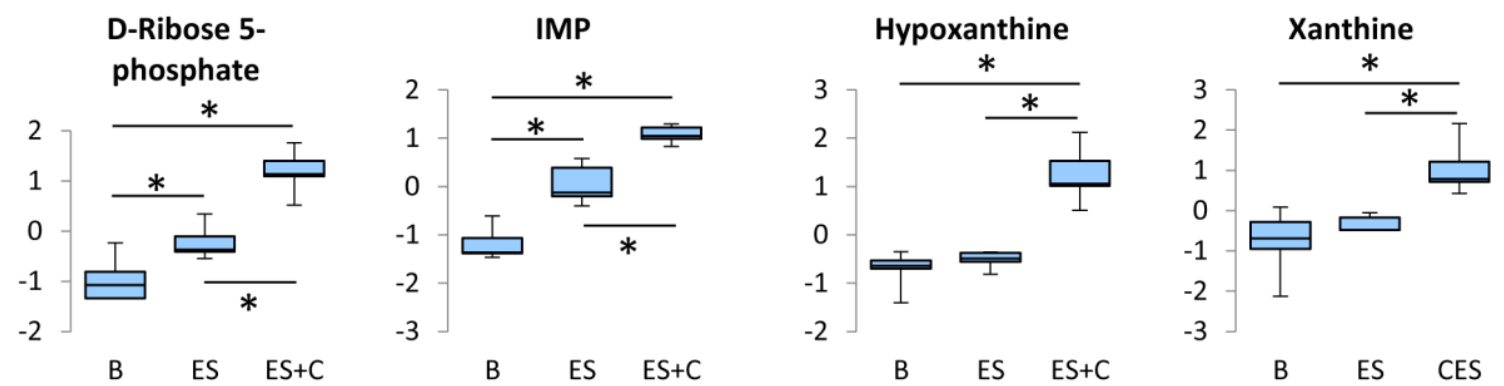

Figure 4. Box plots of the concentration variations of significantly altered metabolites in the pentose phosphate pathway/nucleotide synthesis pathway. Y axes are represented as normalized intensity. The boxes range from the $25 \%$ to the $75 \%$ percentiles. Medians are indicated by horizontal lines within each box. The ends of the whiskers represent the maximum and minimum of the data. * One-way ANOVA with Tukey's post hoc test indicates a significant difference (false discovery rates $(\mathrm{FDR})<0.05$ ) between groups.

\subsection{Acylcarnitine/Tricarboxylic Acid (TCA) Cycle}

Acylcarnitines, which are esters of L-carnitine and fatty acyl-coenzyme A (CoA), are important intermediates in the transport of long-chain fatty acyl-CoA into the mitochondria [27]. Intramitochondrial acylcarnitine is converted back to carnitine and long-chain acyl-CoA by carnitine palmitoyltransferase 2 , which then undergoes $\beta$-oxidation to produce acetyl-CoA. Acetyl-CoA is an essential intermediate metabolite that enters the TCA cycle and is oxidized to yield energy. When the production of short-chain acyl-CoAs exceeds TCA cycle flux, acetyl-CoA is converted to acetylcarnitine by carnitine acetyltransferase [28]. In the present study, caffeine treatment increased O-acetylcarnitine (C2) during skeletal muscle contraction (Figure 5 ), indicating that substrate catabolism during $\beta$-oxidation exceeds the capacity of acetyl-CoA utilization in the TCA cycle.

Muscle contraction-induced AMPK activation inhibits acetyl-CoA carboxylase activity, leading to a decrease in malonyl CoA content [29]. Malonyl CoA is a potent inhibitor of CPT1, an enzyme that combines fatty acyl-CoA with carnitine for transport into the mitochondria for $\beta$-oxidation, and the decrease in malonyl CoA during muscle contraction contributes to the increase in absolute lipid oxidation [30]. In addition, it has been demonstrated that the increase in acetylcarinitine level during muscle contraction decreases the availability of free carnitine, a substrate of CPT1, results in low CPT1 activity [31]. Thus, the accumulation of acetylcarnitine within the skeletal muscle leads to a diminished supply of long-chain fatty acyl-CoA to $\beta$-oxidation [31]. In fact, an increase in acetylcarnitine was observed concomitantly with a decrease in long-chain fatty acid oxidation during exercise in humans [31]. In the present study, L-hexanoyl-carnitine (C6) was reduced by caffeine treatment (Figure 5), indicating that the increase in muscle acetylcarnitine level by caffeine might inhibit $\beta$-oxidation of long-chain fatty acyl-CoA, thereby leading to a decreased supply of short-chain ( C10) fatty acyl-CoA.

However, we found the accumulation of butyrylcarnitine (C4) following caffeine treatment (Figure 5). In accordance with this result, a previous study has demonstrated that the $10 \mathrm{~min}$ of treadmill exercise increased $C 4$ acylcarnitine level in rat skeletal muscle [32]. The authors have suggested that 
the accumulation of $\mathrm{C} 4$ acylcarnitine was attributed to a greater utilization of branched chain amino acids (BCAA: leucine, isoleucine, and valine) [32]. We also found that caffeine treatment increased L-leucine and L-valine levels in contracted muscle (Figure 6). Therefore, it is suggested that the increase in butyrylcarnitine (C4) level originates from the amino acids metabolism.

Succinic acid is a key TCA cycle metabolite, the levels of which can be increased by both long-term exercise training as well as an individual bout of exercise [17,33]. In the present study, caffeine increased succinic acid levels in the contracted muscle (Figure 5). This result suggests that the TCA cycle is activated by caffeine treatment during muscle contraction. However, succinic acid level is reflected by the activity of succinate dehydrogenase, which catalyze succinic acid into fumarate in the TCA cycle. Therefore, to determine the effect of caffeine on the activity level of the TCA cycle during muscle contraction, succinate dehydrogenase level and/or another enzyme activity and metabolite levels need to be investigated.

\section{Acylcarnitine/TCA cycle}
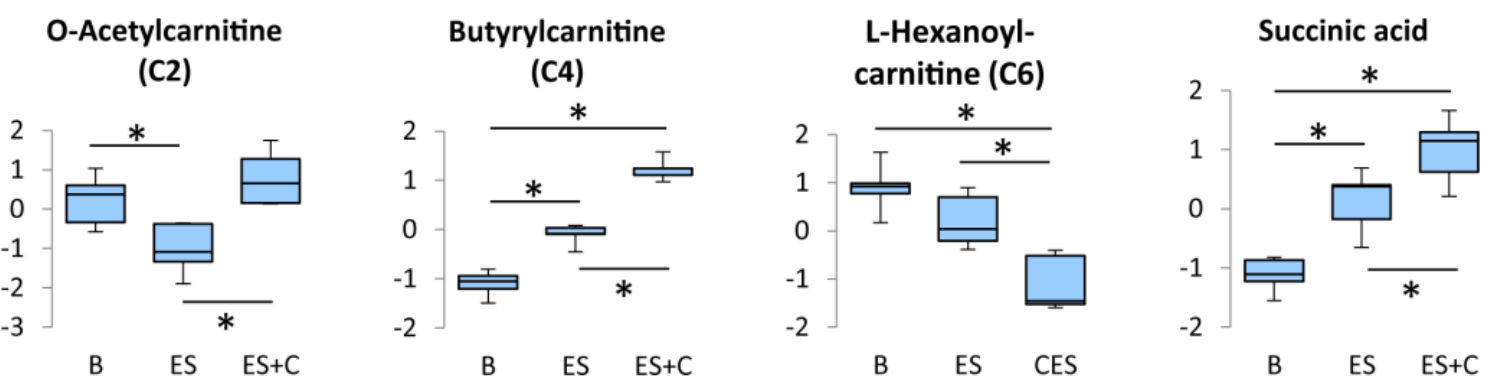

Figure 5. Box plots of the concentration variations of significantly altered metabolites in the Acylcarnitine/ TCA cycle. * One-way ANOVA with Tukey's post hoc test indicates a significant difference (FDR $<0.05$ ) between groups.

In the present study, glycolysis was not affected by caffeine (Figure 8), suggesting that an increase in acetylcarnitine following caffeine treatment can be attributed to the acceleration of acetyl-CoA production from $\beta$-oxidation. Maintaining the acetylcarnitine recycling system is critical for muscle contractile performance and fatigue resistance [34]. Therefore, caffeine may stimulate the acetylcarnitine recycling system, thereby contributing to enhanced muscle endurance capacity. To assess this possibility, further study is required to measure the effects of caffeine on carnitine.

\subsection{Amino Acid/Amino Acid Metabolism}

Protein degradation and subsequent amino acid oxidation contribute slightly to energy supply during exercise as well as to glucose and fatty acid oxidation [35]. Six amino acids are metabolized in the skeletal muscle: BCAA (leucine, isoleucine, and valine), asparagine, aspartate, and glutamate [35]. Leucine can be converted to acetyl-CoA and oxidized in the TCA cycle [36]. The catabolic pathway of valine consists of several enzymatic steps and results in the formation of succinyl-CoA, a member of the TCA cycle. Disruption of BCAA metabolism in skeletal muscle impairs endurance capacity [37]. Thus, the supply of BCAA is considered to be an important factor for controlling exercise metabolism and endurance. In the present study, L-leucine and L-valine were higher in the ES + C group than in the ES group (Figure 6), indicating that these amino acids contribute to energy production of caffeine-treated muscle by incorporated into TCA intermediates.

Aspartate has been suggested to have an ergogenic potential [38]. Aspartate is converted to oxaloacetate by aspartate transaminase, which then enters the TCA cycle. A previous study has demonstrated that the administration of potassium-magnesium-aspartate increased the capacity for prolonged exercise in human [39]. This effect has been supported by other studies [38]. In the present study, caffeine suppressed L-aspartate reduction by ES (Figure 6), indicating that the caffeine-induced 
ergogenic effect may be partly attributed to aspartate preservation during muscle contraction. However, there are a number of negative findings related to aspartate's ergogenic potential [38]. For example, in human volunteers neither the exerted force nor the endurance time increased after oral administration of potassium-magnesium-aspartate [40]. Therefore, further studies are required to clarify the relationship between aspartate and caffeine's ergogenic potential.

Methionine is suggested to be transaminated and is also subjected to transulfuration in the skeletal muscle [41]. Although caffeine increased L-methionine levels (Figure 6) in the contracted muscle, the importance of methionine during exercise is poorly understood.

Alanine is formed from pyruvate and glutamate in the alanine aminotransferase reaction. Increase in skeletal muscle alanine is thought to be due to the enhanced availability of pyruvate and glutamate [42]. Alanine synthesized in the skeletal muscle is released into the blood and taken up by the liver, where it is reconverted into glucose via gluconeogenesis. In the present study, caffeine treatment increased L-alanine during muscle contraction (Figure 6). Considering that pyruvate and glutamate were not changed by caffeine stimulation (Table S1), this caffeine-induced increase in alanine may be attributed to protein degradation. The increase in alanine production may contribute to the increase in $\mathrm{N}$-acetyl-L-alanine levels, which is generated from alanine by phenylalanine $\mathrm{N}$-acetyltransferase, in caffeine and electrically stimulated muscle (Figure 6).

\section{Amino acid/amino acid metabolism}

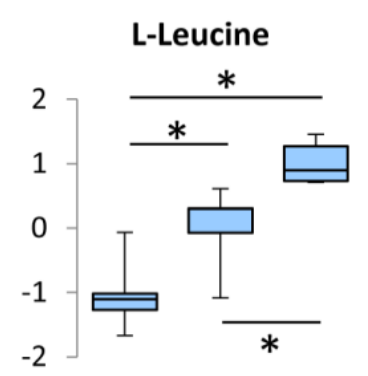

B $\quad \mathrm{ES} \quad \mathrm{ES}+\mathrm{C}$

L-Methionine

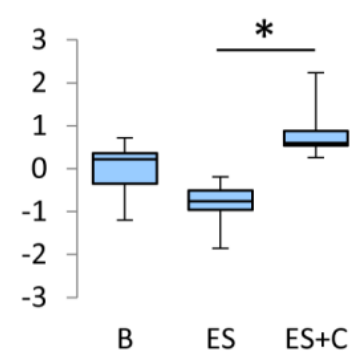

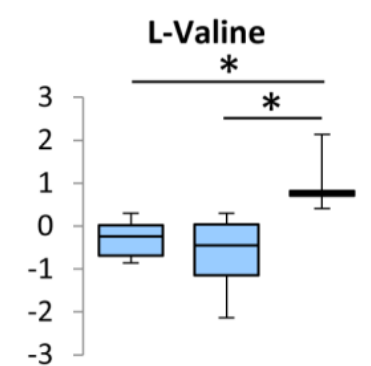

B $\quad$ ES $\quad \mathrm{ES}+\mathrm{C}$

L-Alanine

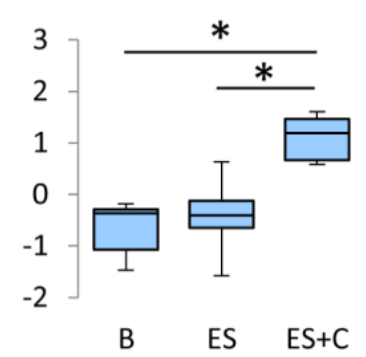

L-Aspartate

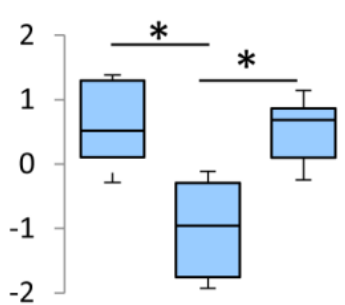

B $\quad \mathrm{ES} \quad \mathrm{ES}+\mathrm{C}$

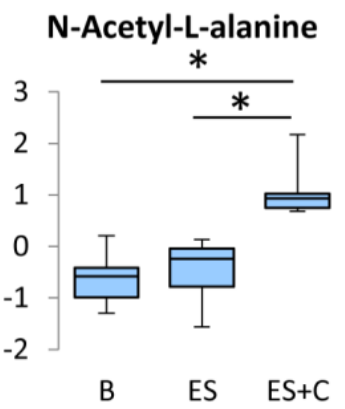

Figure 6. Box plots of the concentration variations of significantly altered metabolites in the amino acid/amino acid metabolism. * One-way ANOVA with Tukey's post hoc test indicates a significant difference $(\mathrm{FDR}<0.05)$ between groups.

\subsection{Others}

N4-acetylcytidine, choline, cytosine, 2-hydroxyisobutyric acid, isatin, guanidinosuccinic acid, oxypurinol, ethylenediaminetetraacetic acid (EDTA), and acetylenedicarboxylate levels were significantly higher in the ES + C group than in the ES group (Figure 7). However, no previous studies have investigated the association between exercise and these metabolites. The functional significance of increased levels of these metabolites in the caffeine-stimulated group needs to be further investigated. 


\section{Others}
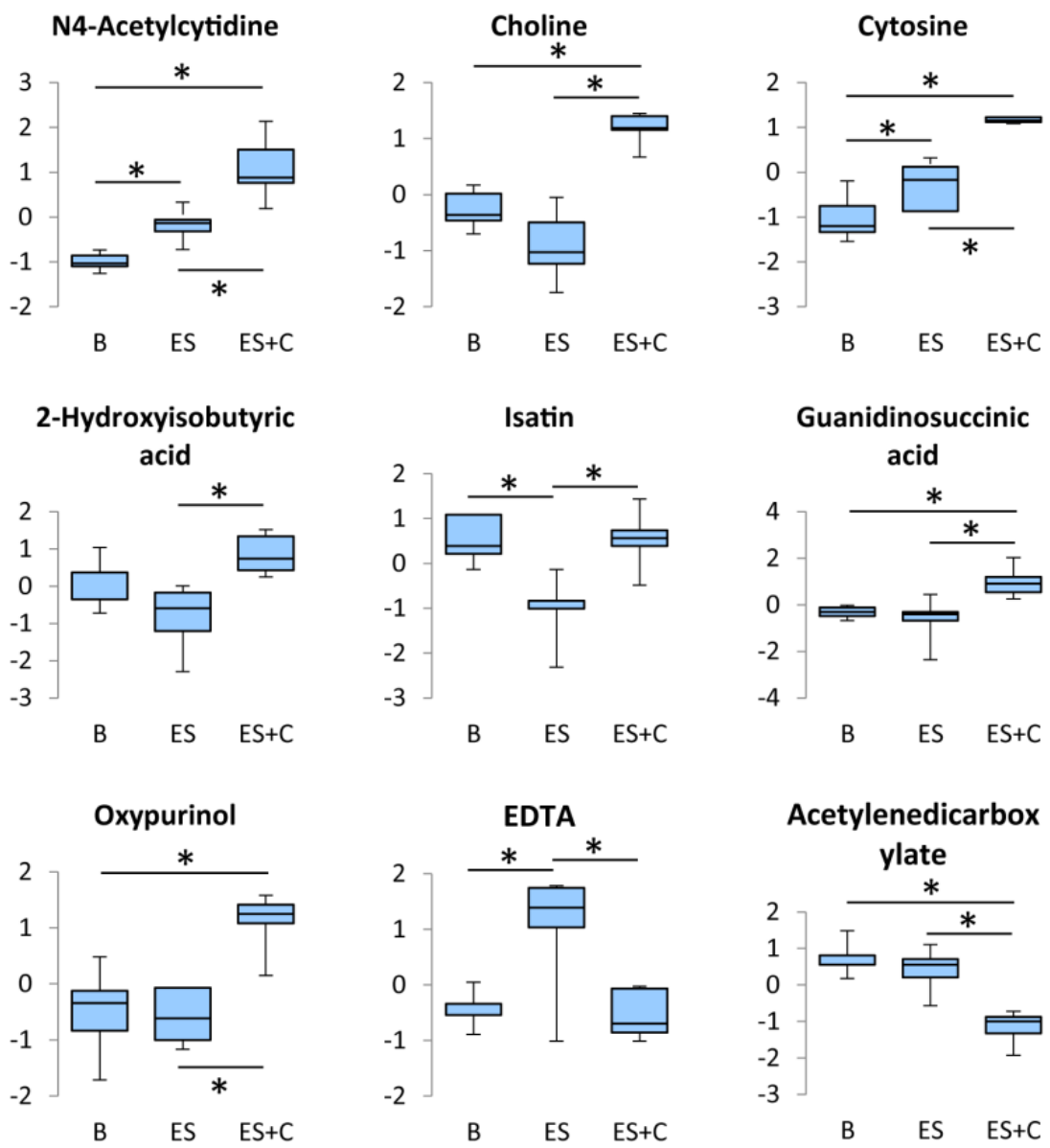

Figure 7. Box plots of the concentration variations of significantly altered metabolites in the other pathways. * One-way ANOVA with Tukey's post hoc test indicates a significant difference $($ FDR $<0.05)$ between groups.

\subsection{Limitations}

Many reactions take place continuously within cells, so concentrations of metabolites are very dynamic. In the present study, we investigated metabolomic responses only 10 min after muscle contraction. Therefore, in a case where increased levels of metabolites are observed, two distinct mechanisms contribute to this observation: either increased production or decreased consumption. For further understanding of caffeine-mediated effects on metabolic changes during muscle contraction, time-course experiments should be conducted.

In this study, we used a concentration of caffeine at $3 \mathrm{mM}$, which would be toxic to humans [43]. Plasma concentration of caffeine after ingestion of $100 \mathrm{mg}$ ( 1 cup of coffee) reaches approximately 5 to $10 \mu \mathrm{M}$ [44], with less than $70 \mu \mathrm{M}$ being the physiological concentrations [45]. Experiments using isolated skeletal muscle preparation have benefits of eliminating the effects of systemic confounders such as circulatory, humoral and neural factors, and of intestinal absorption of caffeine. Taking advantage of this point, a number of studies have unveiled the direct ergogenic properties of caffeine at the supraphysiological concentrations [10,46-49]. Our previous study have demonstrated that $\mu \mathrm{M}$ concentrations were enough to activate AMPK in vivo, but mM concentrations of caffeine were needed to activate AMPK in isolate rat skeletal muscle [5]. Therefore, we should be careful when comparing the results of in vitro and in vivo studies in terms of caffeine concentrations. 
Caffeine is found in foods, beverages, and pharmaceuticals, and the most frequently consumed non-prescription drug. To date, many researchers have discussed the effect of caffeine on energy metabolism and our health [50-52]. However, no study has investigated the caffeine-mediated changes of metabolomic signatures in skeletal muscle and the other organs. Although the present study contributes to unveiling the effect of caffeine on metabolomic responses during muscle contraction condition, the effect of caffeine alone on skeletal muscle metabolism has not been cleared. Further studies are expected to examine the effect of caffeine on muscle's non-contracted condition.

\section{Conclusions}

The present study reveals for the first time that caffeine influences metabolic responses induced by electrically stimulated muscle contraction in isolated rat skeletal muscles. A schematic representation of the metabolic changes induced by caffeine is shown in Figure 8. Many of these changes are related to energy metabolism. First, caffeine promotes contraction-induced activation of the pentose phosphate pathway and increases IMP production. Second, caffeine stimulates $\beta$-oxidation of fatty acyl-CoA, accompanied by increase in acyl-CoA, butylcarnitine and O-acetylcarnitine; however, it does not affect the glycolysis metabolites, glycerol-3-phosphate and L-lactic acid. Third, caffeine increases amino acids levels associated with energy production (L-leucine, L-valine, and L-aspartate). These metabolic alterations induced by caffeine suggest that caffeine accelerates contraction-induced metabolic activations and thereby contributes to muscle endurance performance and exercise benefits to health.

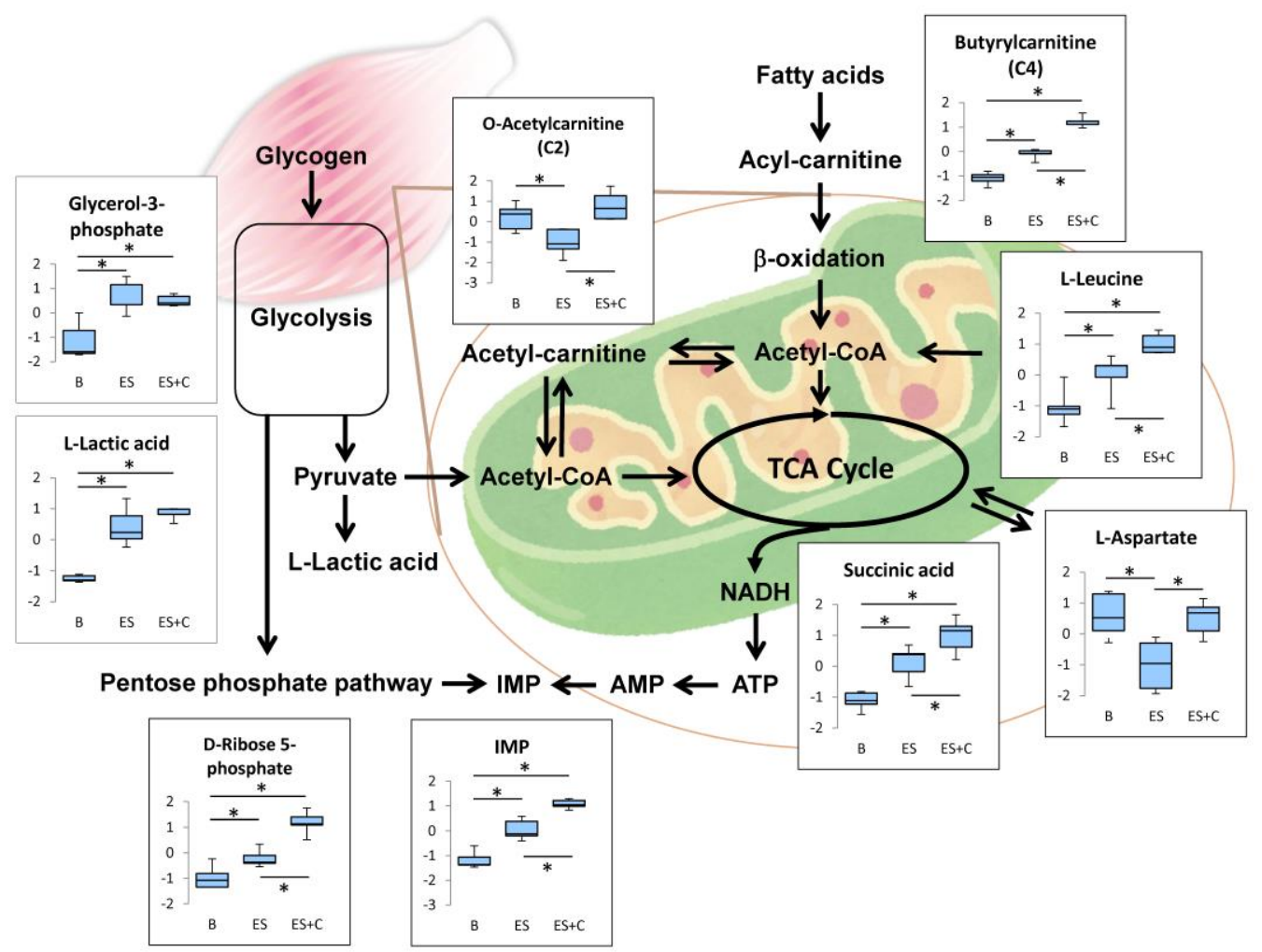

Figure 8. Schematic representation of metabolic pathway changes. * One-way ANOVA with Tukey's post hoc test indicates a significant difference (FDR $<0.05)$ between groups.

Supplementary Materials: The following are available online at http://www.mdpi.com/2072-6643/11/8/1819/s1, Table S1: List of detected metabolites.

Author Contributions: S.T. performed experiments, analyzed the data and contributed with drafting the manuscript. T.H. conceived and designed the research, and drafted the manuscript. T.E. conceived and designed the research, analyzed the data, and drafted the manuscript. All authors interpreted the results, contributed to the discussion, edited and revised the manuscript and read and approved the final version of the manuscript. 
Funding: This study was supported in part by JSPS KAKENHI (Tatsuro Egawa, 18H03148; Tatsuya Hayashi, 19K11520); the Council for Science, Technology and Innovation; SIP (Funding agency: Bio-oriented Technology Research Advancement Institution, NARO) (Tatsuya Hayashi, 14533567). Takeda Research Support (TH, TKDS20170531015).

Conflicts of Interest: The authors declare no conflict of interest.

\section{References}

1. Canto, C.; Auwerx, J. PGC-1alpha, SIRT1 and AMPK, an energy sensing network that controls energy expenditure. Curr. Opin. Lipidol. 2009, 20, 98-105. [CrossRef]

2. Canto, C.; Jiang, L.Q.; Deshmukh, A.S.; Mataki, C.; Coste, A.; Lagouge, M.; Zierath, J.R.; Auwerx, J. Interdependence of AMPK and SIRT1 for metabolic adaptation to fasting and exercise in skeletal muscle. Cell Metab. 2010, 11, 213-219. [CrossRef] [PubMed]

3. Pedersen, B.K.; Febbraio, M.A. Muscles, exercise and obesity: Skeletal muscle as a secretory organ. Nat. Rev. Endocrinol. 2012, 8, 457-465. [CrossRef]

4. Egawa, T.; Hamada, T.; Kameda, N.; Karaike, K.; Ma, X.; Masuda, S.; Iwanaka, N.; Hayashi, T. Caffeine acutely activates $5^{\prime}$ adenosine monophosphate-activated protein kinase and increases insulin-independent glucose transport in rat skeletal muscles. Metab. Clin. Exp. 2009, 58, 1609-1617. [CrossRef]

5. Egawa, T.; Hamada, T.; Ma, X.; Karaike, K.; Kameda, N.; Masuda, S.; Iwanaka, N.; Hayashi, T. Caffeine activates preferentially alpha1-isoform of 5'amp-activated protein kinase in rat skeletal muscle. Acta Physiol. 2011, 201, 227-238. [CrossRef] [PubMed]

6. Tsuda, S.; Egawa, T.; Kitani, K.; Oshima, R.; Ma, X.; Hayashi, T. Caffeine and contraction synergistically stimulate $5^{\prime}$-AMP-activated protein kinase and insulin-independent glucose transport in rat skeletal muscle. Physiol. Rep. 2015, 3, e12592. [CrossRef] [PubMed]

7. Jensen, T.E.; Rose, A.J.; Hellsten, Y.; Wojtaszewski, J.F.; Richter, E.A. Caffeine-induced ca(2+) release increases ampk-dependent glucose uptake in rodent soleus muscle. Am. J. Physiol. Endocrinol. Metab. 2007, 293, E286-E292. [CrossRef] [PubMed]

8. Raney, M.A.; Turcotte, L.P. Evidence for the involvement of camkii and ampk in ca2+-dependent signaling pathways regulating fa uptake and oxidation in contracting rodent muscle. J. Appl. Physiol. 2008, 104, 1366-1373. [CrossRef]

9. Lally, J.S.V.; Jain, S.S.; Han, X.X.; Snook, L.A.; Glatz, J.F.C.; Luiken, J.J.F.P.; McFarlan, J.; Holloway, G.P.; Bonen, A. Caffeine-stimulated fatty acid oxidation is blunted in CD36 null mice. Acta Physiol. 2012, 205, 71-81. [CrossRef] [PubMed]

10. Allen, D.G.; Westerblad, H. The effects of caffeine on intracellular calcium, force and the rate of relaxation of mouse skeletal muscle. J. Physiol. 1995, 487, 331-342. [CrossRef]

11. Konishi, M.; Kurihara, S. Effects of caffeine on intracellular calcium concentrations in frog skeletal muscle fibres. J. Physiol. 1987, 383, 269-283. [CrossRef] [PubMed]

12. Wright, D.C.; Geiger, P.C.; Han, D.H.; Jones, T.E.; Holloszy, J.O. Calcium induces increases in peroxisome proliferator-activated receptor gamma coactivator-1alpha and mitochondrial biogenesis by a pathway leading to p38 mitogen-activated protein kinase activation. J. Biol. Chem. 2007, 282, 18793-18799. [CrossRef] [PubMed]

13. Shen, J.G.; Brooks, M.B.; Cincotta, J.; Manjourides, J.D. Establishing a relationship between the effect of caffeine and duration of endurance athletic time trial events: A systematic review and meta-analysis. J. Sci. Med. Sport 2019, 22, 232-238. [CrossRef] [PubMed]

14. Ribeiro, B.G.; Morales, A.P.; Sampaio-Jorge, F.; Tinoco, F.D.S.; De Matos, A.A.; Leite, T.C. Acute effects of caffeine intake on athletic performance: A systematic review and meta-analysis. Rev. Chil. Nutr. 2017, 44, 283. [CrossRef]

15. Southward, K.; Rutherfurd-Markwick, K.J.; Ali, A. The Effect of Acute Caffeine Ingestion on Endurance Performance: A Systematic Review and Meta-Analysis. Sports Med. 2018, 48, 1913-1928. [CrossRef] [PubMed]

16. Dotzert, M.S.; Murray, M.R.; McDonald, M.W.; Olver, T.D.; Velenosi, T.J.; Hennop, A.; Noble, E.G.; Urquhart, B.L.; Melling, C.W.J. Metabolomic Response of Skeletal Muscle to Aerobic Exercise Training in Insulin Resistant Type 1 Diabetic Rats. Sci. Rep. 2016, 6, 26379. [CrossRef] [PubMed] 
17. Starnes, J.W.; Parry, T.L.; O’Neal, S.K.; Bain, J.R.; Muehlbauer, M.J.; Honcoop, A.; Ilaiwy, A.; Christopher, P.M.; Patterson, C.; Willis, M.S. Exercise-Induced Alterations in Skeletal Muscle, Heart, Liver, and Serum Metabolome Identified by Non-Targeted Metabolomics Analysis. Metabolites 2017, 7, 40. [CrossRef] [PubMed]

18. Nesher, R.; Karl, I.E.; Kaiser, K.E.; Kipnis, D.M. Epitrochlearis muscle. I. Mechanical performance, energetics, and fiber composition. Am. J. Physiol. 1980, 239, E454-E460. [CrossRef]

19. Zetan, N.; Wallberg-Henriksson, H.; Henriksson, J. The rat epitrochlearis muscle: Metabolic characteristics. Acta Physiol. Scand. 1988, 134, 155-156. [CrossRef]

20. Miyamoto, L.; Egawa, T.; Oshima, R.; Kurogi, E.; Tomida, Y.; Tsuchiya, K.; Hayashi, T. AICAR stimulation metabolome widely mimics electrical contraction in isolated rat epitrochlearis muscle. Am. J. Physiol. Physiol. 2013, 305, C1214-C1222. [CrossRef]

21. Larsson, O.; Wahlestedt, C.; Timmons, J.A. Considerations when using the significance analysis of microarrays (SAM) algorithm. BMC Bioinform. 2005, 6, 129. [CrossRef] [PubMed]

22. Stincone, A.; Prigione, A.; Cramer, T.; Wamelink, M.M.; Campbell, K.; Cheung, E.; Olin-Sandoval, V.; Gruning, N.M.; Kruger, A.; Tauqeer Alam, M.; et al. The return of metabolism: Biochemistry and physiology of the pentose phosphate pathway. Biol. Rev. Camb. Philos. Soc. 2015, 90, 927-963. [CrossRef] [PubMed]

23. Dodd, S.L.; Johnson, C.A.; Fernholz, K.; Cyr, J.A. The role of ribose in human skeletal muscle metabolism. Med. Hypotheses 2004, 62, 819-824. [CrossRef] [PubMed]

24. Hellsten, Y.; Richter, E.A.; Kiens, B.; Bangsbo, J. AMP deamination and purine exchange in human skeletal muscle during and after intense exercise. J. Physiol. 1999, 520, 909-920. [CrossRef] [PubMed]

25. Mahoney, D.E.; Hiebert, J.B.; Thimmesch, A.; Pierce, J.T.; Vacek, J.L.; Clancy, R.L.; Sauer, A.J.; Pierce, J.D. Understanding D-Ribose and Mitochondrial Function. Adv. Biosci. Clin. Med. 2018, 6, 1-5. [CrossRef]

26. Palić, I.R.; Đorđević, A.S.; Ickovski, J.D.; Kostic, D.A.; Dimitrijevic, D.S.; Stojanović, G.S. Xanthine Oxidase: Isolation, Assays of Activity, and Inhibition. J. Chem. 2015, 2015, 1-8.

27. Schooneman, M.G.; Vaz, F.M.; Houten, S.M.; Soeters, M.R. Acylcarnitines: Reflecting or inflicting insulin resistance? Diabetes 2013, 62, 1-8. [CrossRef]

28. Furuichi, Y.; Goto-Inoue, N.; Fujii, N.L. Role of carnitine acetylation in skeletal muscle. J. Phys. Fit. Sports Med. 2014, 3, 163-168. [CrossRef]

29. Hardie, D.G.; Pan, D.A.; Hardie, G. Regulation of fatty acid synthesis and oxidation by the AMP-activated protein kinase. Biochem. Soc. Trans. 2002, 30, 1064-1070. [CrossRef]

30. Miura, S.; Tadaishi, M.; Kamei, Y.; Ezaki, O. Mechanisms of exercise- and training-induced fatty acid oxidation in skeletal muscle. J. Phys. Fit. Sports Med. 2014, 3, 43-53. [CrossRef]

31. Kiens, B. Skeletal Muscle Lipid Metabolism in Exercise and Insulin Resistance. Physiol. Rev. 2006, 86, $205-243$. [CrossRef]

32. Overmyer, K.A.; Evans, C.R.; Qi, N.R.; Minogue, C.E.; Carson, J.J.; Chermside-Scabbo, C.J.; Koch, L.G.; Britton, S.L.; Pagliarini, D.J.; Coon, J.J.; et al. Maximal oxidative capacity during exercise is associated with skeletal muscle fuel selection and dynamic changes in mitochondrial protein acetylation. Cell Metab. 2015, 21, 468-478. [CrossRef]

33. Huffman, K.M.; Koves, T.R.; Hubal, M.J.; Abouassi, H.; Beri, N.; Bateman, L.A.; Stevens, R.D.; Ilkayeva, O.R.; Hoffman, E.P.; Muoio, D.M.; et al. Metabolite signatures of exercise training in human skeletal muscle relate to mitochondrial remodelling and cardiometabolic fitness. Diabetologia 2014, 57, 2282-2295. [CrossRef]

34. Seiler, S.E.; Koves, T.R.; Gooding, J.R.; Wong, K.E.; Stevens, R.S.; Ilkayeva, O.R.; Wittmann, A.H.; DeBalsi, K.L.; Davies, M.N.; Lindeboom, L.; et al. Carnitine Acetyltransferase Mitigates Metabolic Inertia and Muscle Fatigue During Exercise. Cell Metab. 2015, 22, 65-76. [CrossRef]

35. Wagenmakers, A.J.M. 11 muscle amino acid metabolism at rest and during exercise: Role in human physiology and metabolism. Exerc. Sport Sci. Rev. 1998, 26, 287-314. [CrossRef]

36. Zhang, S.; Zeng, X.; Ren, M.; Mao, X.; Qiao, S. Novel metabolic and physiological functions of branched chain amino acids: A review. J. Anim. Sci. Biotechnol. 2017, 8, 10. [CrossRef]

37. She, P.; Zhou, Y.; Zhang, Z.; Griffin, K.; Gowda, K.; Lynch, C.J. Disruption of bcaa metabolism in mice impairs exercise metabolism and endurance. J. Appl. Physiol. 2010, 108, 941-949. [CrossRef]

38. Trudeau, F. Aspartate as an Ergogenic Supplement. Sports Med. 2008, 38, 9-16. [CrossRef]

39. Ahlboro, B.; Ekelund, L.-G.; Nilsson, C.-G. Effect of Potassium-Magnesium-Aspartate on the Capacity for Prolonged Exercise in Man. Acta Physiol. Scand. 1968, 74, 238-245. [CrossRef] 
40. de Haan, A.; van Doorn, J.E.; Westra, H.G. Effects of potassium + magnesium aspartate on muscle metabolism and force development during short intensive static exercise. Int. J. Sports Med. 1985, 6, 44-49. [CrossRef]

41. Scislowski, P.W.D.; Hokland, B.M.; Thienen, W.I.A.D.-V.; Bremer, J.; Davis, E.J. Methionine metabolism by rat muscle and other tissues. Occurrence of a new carnitine intermediate. Biochem. J. 1987, 247, 35-40. [CrossRef]

42. Ishikura, K.; Ra, S.-G.; Ohmori, H. Exercise-induced changes in amino acid levels in skeletal muscle and plasma. J. Phys. Fit. Sports Med. 2013, 2, 301-310. [CrossRef]

43. Fredholm, B.B.; Bättig, K.; Holmén, J.; Nehlig, A.; Zvartau, E.E. Actions of caffeine in the brain with special reference to factors that contribute to its widespread use. Pharmacol. Rev. 1999, 51, 83-133.

44. Derungs, A.; Donzelli, M.; Berger, B.; Noppen, C.; Krahenbuhl, S.; Haschke, M. Effects of cytochrome p450 inhibition and induction on the phenotyping metrics of the basel cocktail: A randomized crossover study. Clin. Pharmacokinet. 2016, 55, 79-91. [CrossRef]

45. Graham, T.E. Caffeine and exercise: Metabolism, endurance and performance. Sports Med. 2001, 31, 785-807. [CrossRef]

46. Rossi, R.; Bottinelli, R.; Sorrentino, V.; Reggiani, C. Response to caffeine and ryanodine receptor isoforms in mouse skeletal muscles. Am. J. Physiol. Physiol. 2001, 281, C585-C594. [CrossRef]

47. Huddart, H.; Abram, R.G. Modification of excitation-contraction coupling in locust skeletal muscle induced by caffeine. J. Exp. Zool. 1969, 171, 49-58. [CrossRef]

48. Weber, A.; Herz, R. The Relationship between Caffeine Contracture of Intact Muscle and the Effect of Caffeine on Reticulum. J. Gen. Physiol. 1968, 52, 750-759. [CrossRef]

49. Luttgau, H.C.; Oetliker, H. The action of caffeine on the activation of the contractile mechanism in straited muscle fibres. J. Physiol. 1968, 194, 51-74. [CrossRef]

50. Nawrot, P.; Jordan, S.; Eastwood, J.; Rotstein, J.; Hugenholtz, A.; Feeley, M. Effects of caffeine on human health. Food Addit. Contam. 2003, 20,1-30. [CrossRef]

51. Grosso, G.; Godos, J.; Galvano, F.; Giovannucci, E.L. Coffee, Caffeine, and Health Outcomes: An Umbrella Review. Annu. Rev. Nutr. 2017, 37, 131-156. [CrossRef]

52. Harpaz, E.; Tamir, S.; Weinstein, A. The effect of caffeine on energy balance. J. Basic Clin. Physiol. Pharmacol. 2017, 28, 1-10. [CrossRef]

(C) 2019 by the authors. Licensee MDPI, Basel, Switzerland. This article is an open access article distributed under the terms and conditions of the Creative Commons Attribution (CC BY) license (http://creativecommons.org/licenses/by/4.0/). 